\title{
Zur Pathogenese der netzförmigen Livedo bei Tuberkulose.
}

\author{
Von \\ Dr. Friedrich Fischl, \\ Assistent.
}

(Aus der II. dermatologischen Abteilung des allgemeinen Krankenhauses in Wien. [Vorstand: Prof. Dr. Ehrmann].)

Mit 1 Textabbildung.

(Eingegangen am 15. August 1921.)

Unter Livedo lenticularis versteht $H$. G. Adams on, der diese Nomenklatur in die Literatur gebracht hat, eine hauptsächlich an den Unterschenkeln auftretende, netzförmige Marmorierung der Haut, und zwar derart, daß bläulichrote Maschen von ca. $3 \times 6 \mathrm{~mm}$ Breite weiße Hautfelder von ca. Heller- bis Zwanzighellerstückgröße einschließen. Manchmal ist dieses Umfaßtwerden der weißen Hautpartien durch das bläuliche Maschenwerk kein vollständiges. Auch Schwinden einzelner Äste des hyperämischen Maschenwerkes kommt vor, was sich in einer Vergrößerung derweißen Innenfläche auf das Mehrfache der erwähnten Maße äußert. Ätiologisch können verschiedene Ursachen diese eigenartige Projektion eines pathologischen Vorganges auf die Haut hervorrufen. Ehr mann war wohl der erste, der 1906 unter dem Namen Livedo racemosa bei Syphilis das Bild klinisch festgesetzt hat und dessen pathologisch-anatomisches Substrat, eine Endarteriitis der kleinen Arterien des tiefen, zwischen Cutis und Subeutis verlaufenden Gefäßnetzes erkannte. Er beobachtete es bei Luetikern des späteren Sekundär- und des Tertiärstadiums insbesondere bei solchen, deren Gefäßsystem durch chronische Noxen, hauptsächlich Alkohol geschädigt war, ferner bei seniler oder vorzeitiger Arteriosklerose. Dieses Gefäßphänomen wird durch therapeutische Eingriffe gewöbnlich (bis auf einen Fall $F$ h $r$ man $n \mathrm{~s}$ ) fast gar nicht beeinflußt, eine Tatsache, die deswegen hervorzuheben ist, da C. Sch midt eine ähnliche bläulichviolette Gefäßzeichnung an der Haut eines Luetikers des frühen Sekundärstadiums beschreibt, die auf spezifische Therapie rasch und völlig schwand. Auch der histologische Befund, den der genannte Autor bei seinem Falle erheben konnte, ist von den durch Ehr mann in allen seinen Fällen festgestellten anatomischen Veränderungen ganz verschieden, Sohmidt stellte nämlich im wesentlichen eine Endo- und Periphlebitis der tieferen Venen fest, die eine venöse Stase des oberflächlichen Gefäßnetzes hervorrufen, was sich eben in Form der genannten livedoähnlichen Zeichnung auf der Haut ausdrückt; die Arterien fand er völlig intakt. Stellt man sich zur Frage, ob der gennante klinisch und histologisch beschriebene Fall sich mit der von Ehrmann beschriebenen Livedo racemosa deckt, kritisch, so kann dies mit Sicherheit aus den oben angeführten Gründen (pathologisch-anatomischer Befund, therapeutische Beeinflussung) in Abrede gestellt werden. Es dürfte sich da wohl um eine spezifische Erkrankung der Venen im Frühstadium der Lues, die ja nicht allzu selten ist, gehandelt haben, die im Erythema nodosum lueticum besonders stark ausgeprägt ist, was durch Levaditi-Spirochäten-Schnittfärbung wohl auch zu beweisen ge- 
wesen wäre. Wesentlich schwieriger ist die Beurteilung der Befunde, die Ale xander ebenfalls klinisch und histologisch an zwei nicht luetischen Patienten erhoben hat und die bis auf etwas atypische Lokalisation (Brust, Bauch, Rücken, Glutealgegend) in ihrem Aspekte ganz an die Fälle Ehrmanns bis auf die Verschmälerung der baumförmigen Zeichnung erinnern, jedoch pathologisch anatomisch zu keinerlei verwertbarem Ergebnis geführt haben. Es konnten nämlich keine Veränderungen am arteriellen oder venösen GefäBsystem gefunden werden. Das histologische Bild zeigte nur geringe, hauptsächlich aus Lymphocyten, spärlichen polynucleären Leukocyten und ganz vereinzeltem Plasma, sowie Mastzellen zusammengesetzte Infiltratmäntel um die prall gefüllten Gefäße des papillaren Körpers und der Cutis.

Wir entnehmen den angeführten Beobachtungen, daß nicht nur die Lues das Phänomen der Livedo racemosa auszulösen imstande ist, und in einer jüngst erschienenen Arbeit von Hess und Kerl wird die Frage vom internen und dermatologischen Standpunkt an der Hand einiger sehr sorgsam insbesondere klinisch genauest untersuchter Fälle studiert. Als Frgebnis kann die Feststellung angesehen werden, daß nicht nur exogene Noxen, wie Lues, Alkoholismus und Tuberkulose den sechs Fällen Adamsons von Livedo lenticularis bei Erythema induratum Bazin wurde einer eigener Beobachtung hinzugefügt - in die Gruppe der Livedo racemosa gehörige Hauterscheinungen auslösen können, sondern, wie die interessanten Beobachtungen Gros z und Kerls ergeben, dasselbe Symptomenbild auch durch schwere arteriosklerotische Prozesse der Gefäße hervorgerufen werden kann. Schließlich fügen Hess und Kerl noch das in der Veranlagung gegebene Moment der Abnormitäten des Gefäßsystems an der Hand eigener Untersuchungen (die von Wolkow geschilderte Gefäßverdickung an der A. radialis Jugendlicher, die vermehrten Reflexe der Netzhautarterien derartiger verschieden stigmatisierter Individuen) als kausale Grundlage hinzu. Schließlich wäre noch der jüngst erschienenen Arbeit Waels ch" über dieses Thema", Über Livedo racemosa bei Tuberkulose" zu gedenken. Dieser Autor beschreibt klinisch 3 Fälle, bei denen das Syndrom Lungentuberkulose, Tuberkulide, Livedo racemosa zu verzeichnen ist. Nur ein Fall konnte histologisch untersucht werden, und dieser ergab einen sehr dürftigen Befund, nämlich Gefäßerweiterung im Stratum subpapillare und geringe perivasculare Infiltration. Der Anschauung Waels ch', es handle sich bei der Entstehung der Livedo racemosa um eine Beeinflussung des Venenwand selbst, kann wohl zumindest in dieser allgemeinen Form nicht beigepflichtet werden, da ja Veränderungen an den Venen weder von ihm noch von Alexander nachgewiesen werden konnten, die positiven, ständig wieder erhobenen endarteriitischen Veränderungen in den Fällen Ehrmanns aber bei der Livedo racemosa der Luetiker im pathogenetischen Sinne gedeutet werden müssen. Ob der bei tuberkulösen Individuen beobachteten Livedo racemosa-ähnlichen Zeichnung nicht ein anderes pathologisch-anatomisches Substrat zugrunde liegt, muß weiteren Untersuchungen vorbehalten bleiben; daß es sich in allen diesen Fällen um klinisch sehr ähnliche Bilder handle, ist wohl sicher, hingegen lassen Differenzen in den klinischen (Breite der Maschen) und pathologisch-anatomischen Befunden - Ehrmanns Endarteriitis in allen seiner Luesfälle gegenüber dem negativen Ergebnis der histologischen Untersuchungen des GefäBsystems bei Alexander, Hess und Kerl — daran denken, daß wir es nicht mit derselben Erscheinung zu tun haben, wenn auch deren klinischer Symptomenkomplex sehr ähnlich ist. Wie erwähnt, hat Ad a ms on wohl eine von ihm Livedo lenticularis genannte Gefäßzeichnung bei einigen Fällen von Ery. thema induratum Bazin beobachtet und auch Hess und Kerl beschreiben einen derartigen Fall. Da der erstgenannte Autor keinen histologischen Befund erbrachte, die histologische Untersuchung der letzterwähnten Untersucher aber nur eine geringe Erweiterung der Gefäßlumina der subpapillaren Schicht mit geringer 
Infiltration um dieselbe, Ödem des Coriums und in den tieferen Schichten spärliches perivasculäres Infiltrat bei fehlender Infiltration des Fettgewebes, der Subcutis, in der sich eine ganz thrombosierte Vene fand, ergab, sei es gestattet, ohne auf die Krankengeschichten zweier selbst beobachteter Fälle von Livedo lenticularis bei Erythema induratum Bazin näher einzugehen (siehe Abbildung), die von uns fest. gestellten histologischen Befunde wiederzugeben.

Aus der Anamnese von Fall I, St. B., 21 Jahre alt, Prot. Nr. 8910/1921, ist hervorzuheben, daß Pat. nach ihrer Angabe die gegenwärtige Affektion (Lymphomata colli, Erythema induratum Bazin, papulonekrotische Tuberkulide der Unter-

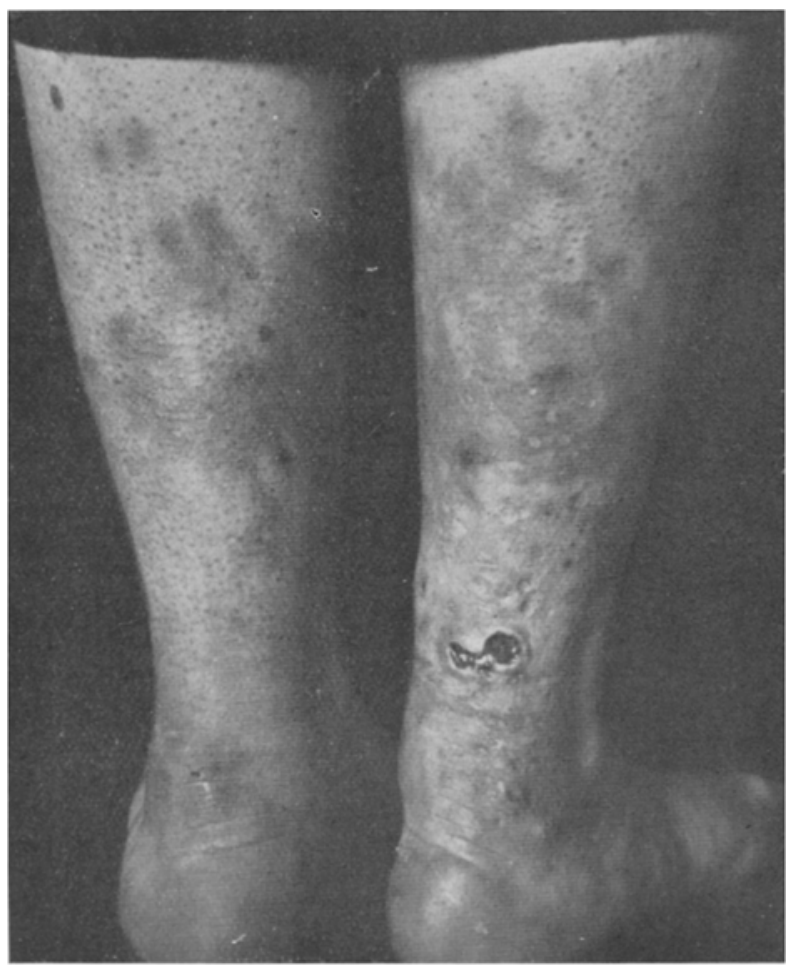

Abb. 1.

schenkel und Livedo racemosa-ähnliche Zeichnung daselbst) seit ca 3-4 Jahrer hat; das Leiden tritt wohl im Winter stärker auf, ist aber zur Zeit der Spitalsaufnahme (Ende März) besonders zutage getreten. Die Lungenuntersuchung ergab einen obsoleten rechtsseitigen Spitzenprozeß, Moro-Reaktion stark positiv, ebenso Pirquet-Reaktion.

Bei Fall II, L. St., 29 Jahre alt, ambulant 21. IV. 1921, ergibt die Anamnese, daß die heute noch bestehenden Lymphomata colli bereits vor 20 Jahren auftraten und daß im 14. Lebensjahre Knötchen auf der Streckseite der Arme und Beine sich hinzugesellten, aus denen sich Eiter entleerte; die genannten Knötchen trockneten ein und heilten erst nach Monaten unter ,Blatternarben" aus. Die knotenförmigen Infiltrate an den Unterschenkeln, sowie die eigenartige Gefäßzeichnung besteht erst seit drei Monaten (Jänner 1921). 
Aus dem internen Status wäre ein röntgenologisch festgestellter verkalkter Herd im Oberlappen der rechten Lunge zu erwähnen. Der dermatologische Befund ergab stellenweise perforierte Knoten vom Erythema induratum Bazin, papulonekrotische Tuberkulide der Streckseiten der Arme und Beine, zum Teil Narben nach abgeheilten derartigen Efflorescenzen. Pirquet-Reaktion positiv, MoroReaktion sehr stark positiv (über die einzelnen handflächengroße) Knötchen größtenteils konfluierend und nekrotisch).

Histologische Befunde: Fall 1. Epidermis intakt, starke Erweiterung der subpapillaren Gefäße und bedeutende Rundzelleninfiltration um dieselben. Ödem des Coriums und bis in dasselbe reichende Infiltration um die Gefäße. An einer Stelle, offenbar dem Querschnitt einer Masche der Livedo entsprechend, diffuse Infiltration des Gewebes von der Papillarschicht bis an die Subcutis mit Rundzellen sowie spärlichen Leukocyten. Senkrecht abwärts von dieser Stelle Konfluenz der Zellen des subcutanen Fettgewebes zu großen Räumen infolge Auflösung der Zwischenwände. Dichte Infiltration des interstitiellen Gewebes zwischen den Fezttellen vom Rande her mit epitheloiden Zellen, Leukocyten und zahlreichen typischen Langhansschen Riesenzellen, die der Achse der angegebenen Veränderung entsprechen. Die an der Grenze von Cutis und Suboutis liegenden Venen (das tiefe Gefäßnetz) zeigen auf Serienschnitten streckenweise eine Peri- und Endophlebitis, erstere schwindet, während die Periphlebitis sie weiter begleitet. Die Endophlebitis ist gekennzeichnet durch eine Intimawucherung, die in das Lumen knospenartig vorspringt, die Periphlebitis durch eine geringe Rundzelleninfiltration um die Gefäße der Adventitia und der weiteren Umgebung derselben äußert. Die Elasticafärbung (Orcein-Methylenblau) zeigt ein Verwaschensein der elastischen Fasern der befallenen Gefäßwände und das Fehlen derselben im Bereiche der infiltrierten Papillarschicht, Cutis und Subcutis.

Fall II. Epidermis normal. Geringe Rundzelleninfiltration um die erweiterten subpapillaren Venen, starkes Ödem des Coriums. Die Venen der Grenze zwischen Corium und Subcutis sind großenteils in ihrem Lumen durch eine dasselbe verengende Intimawucherung charakterisiert. Das Fettgewebe zeigt eine ähnliche interstitielle Infiltration wie der erstgenannte Fall mit Epitheloidzellen, Lymphocyten und Riesenzellen. Die Veränderungen, welche hier bei der Iivedo der Tuberkulösen klinisch beobachtet wurden, lassen zunächst erkennen, daß das Astwerk derselben etwas komplizierter ist als bei der von Ehrmann beschriebenen Livedo racemosa, die mit Erkrankung der Arterien einhergeht. Die Äste sind breiter und zeigen nicht die axiale Erhöhung, wenigstens nicht in dem Maße. Histologisch sehen wir eine Peri- und Endophlebitis (vermutungsweise ist die von $\mathrm{Kerl}$ und Hess beschriebene Thrombosierung der Venen auch durch einen sog. organisierten Thrombus, i. e. Endophlebitis bedingt). In beiden unserer Fälle besteht eine Affektion der 
axialen Vene des befallenen Streifens etwas unterhalb der Grenze der Cutis und Subcutis in den breiten Septen des vielfach spezifisch veränderten Fettgewebes. Auch um die von der Tiefe aufsteigenden Arterien findet man hier und da Infiltration. Das Ganze stellt gewissermaßen ein nicht völlig zur Reife gekommenes Erythema induratum Bazin vor, bei dem ja Endophlebitiden neben Endarteriitiden vorkommen. Es ist übrigens nicht ausgeschlossen, daß in der Tiefe des Gewebes, an der Fascie noch tiefergreifende Veränderungen, die man nur bei Excisionen, die bis an die Fascie reichen, zu Gesicht bekommt, sich vorfinden.

Soweit wir bei der geringen Kenntnis der Venenpathologie sagen können, handelt es sich hier um eine Stauung, bedingt durch Verengerung der Venen des tiefen Gefäßnetzes; möglicherweise gehen auch noch Venenspasmen der anatomischen Veränderung voraus. Durch diese Okklusion staut sich das aus dem venösen Teil der Capillaren abzuführende Blut in den Capillarschlingen. Der Umstand, daß hier nicht die Zufuhr des Blutes durch die Arterie gedrosselt ist, sondern bloß der Abfluß gehemmt ist, bewirkt, daß eine größere Menge Blutes sich in den Capillaren stauen kann. Die Folge ist, daß die mit angestautem Blut gefüllten Hautpartien breiter erscheinen als in jenen Fällen, wo die Arterie verengert ist. Auch der Druck, den das tuberkulöse Infiltrat in den Interstitien des Fettgewebes auf die daselbst befindlichen Venen ausübt, dürfte zur Kompression des tiefen Netzes wohl beitragen.

Den klinischen und den pathologisch-histologischen Befunden ist also zu entnehmen, daß das der Livedo racemosa bis zu einem gewissen Grade ähnliche Bild der Livedo lenticularis bei tuberkulösen Individuen (Tuberculosa pulmonum, Erythema induratum Bazin, papulo-nekrotische Tuberkulide, Lymphomata colli) vorkommt, was gegen die ätiologische Eindeutigkeit der Livedo (Lues) spricht. Ihr ausschließliches Vorkommen bei Syphilitikern wurde übrigens von Ehrmann nicht in Anspruch genommen; er selbst wies darauf hin, daß krampfartige Zustände der Arterien ähnliche klinische Erscheinungen hervorrufen können.

1. Das histologische Bild der Livedo lenticularis ist durch ein tuberkulöses Gewebe mit Epitheloidzellen, Riesenzellen, Leukocyten im interstitiellen Fettgewebe der Subcutis, sowie durch Endo- und Periphlebitis (Tuberkulotoxine?) der tiefen Venen, Erweiterung des oberflächlichen subpapillaren Gefäßnetzes und Rundzelleninfiltration um dieselben gekennzeichnet.

2. Die Livedo racemosa bei Lues unterscheidet sich von den Fällen mit tuberkulöser Ätiologie, weil bei letzteren die Endophlebitis im Vordergrunde steht; so unterscheidet sich auch der Fall Schmidts 
von den Fällen Ehrmanns, weil bei ihm eine Endophlebitis gefunden wurde.

3. Die Livedo lenticularis (Adamson) stellt gewissermaßen ein nicht völlig zur Reife gekommenes Erythema induratum Bazin dar.

\section{Literatur.}

Ada ms on, H. G., Livedo lenticularis. Brit. med. journ of derm. 1916, Nr. 10-12, S. 291. Ref. Derm. Zeitschr. 25, 214. 1918. - Alexander, Arthur, Über Livedo racemosa ohne nachweisbare Lues. Derm. Wochenschr. 6r, 479. 191ع. - Bruh ns, C., Berl. derm. Ges. Sitz. vom 15. I. 1918, ref. Derm. Wochenschr. 1918 Nr. 14. - Ehrmann, S., Mitteilung über syph. Veränderungen der Hautgefäße Kongr. d. dtschen Gesellsch. f. inn. Med. 1905. - E Er man n, S., Weitere Mitteilungen über syphilitische Veränderungen der Hautgefäße. Arch. f. Dermatol. u. Syphilis, 113, 261. 1913 - Ehr man n, S., Zur Frage der Livedo racemosa. Derm. Wochenschr. 69. 1919. - Ehrmann, S., Differentialdiagnostischer Atlas der Hautkrankheiten und der Syphilide. 1912. Kapitel über Livedo racemosa. Grosz, S., Wien. derm. Ges., Ber. noch nicht veröffentlicht. - Hess und Kerl, W., Über die Pathogenese der Livedo racemosa und ihr nahestehender Hautveränderungen. Derm. Zeitschr. (noch nicht ersehienen). - Sch midt, K., Zur Kenntnis des Ehrmannschen Luesphänomens. Arch. f. Dermatol. u. Syphilis 114, 191. 1913. - Waelsch, L., Über Livedo racemosa bei Tuberkulose. Arch. f. Derm. u. Syphilis 132, 452. 1921. 